\title{
Effects of combined endurance and resistance training in Amyotrophic Lateral Sclerosis: A pilot, randomized, controlled study
}

\author{
Antonio Merico (1), Marianna Cavinato (1), Caterina Gregorio (2), Alessandra Lacatena \\ (3), Elisabetta Gioia (4), Francesco Piccione (1), Corrado Angelini (1) \\ (1) Neurorehabilitation Department, San Camillo Hospital Foundation, Institute of Care and \\ Research, Venice. Italy; (2) Department of Economic, mathematical and statistical sciences, \\ University of Trieste, Italy; (3) Rehabilitation Department, Azienda Sanitaria Locale, Taranto, \\ Italy; (4) Rehabilitation Department, Unità Locale Socio-Sanitaria Serenissima, Venice, Italy
}

This article is distributed under the terms of the Creative Commons Attribution Noncommercial License (CC BY-NC 4.0) which permits any noncommercial use, distribution, and reproduction in any medium, provided the original author(s) and source are credited.

\begin{abstract}
Based on available evidence, muscle strengthening and cardiovascular exercises can help maintain function and not adversely affect the progression of disease in patients with ALS. However, this evidence is not sufficiently detailed to recommend a specific exercise prescription. The purpose of this project was to assess clinical outcomes of a combined exercise programme to increase knowledge of rehabilitation in ALS patients. 38 ALS patients were assigned randomly to two groups: one group underwent a specific exercise programme (ALS-EP) based on a moderate aerobic workout and isometric contractions, and the second group followed a standard neuromotor rehabilitation treatment. Objective evaluation consisted of cardiovascular measures, muscle strength and fatigue. Some positive effects of physical activity on ALS patients were found. Among the benefits, an overall improvement of functional independence in all patients, independently of the type of exercise conducted was seen. In addition, improvements in muscle power, oxygen consumption and fatigue were specifically observed in the ALS-EP group, all hallmarks of a training effect for the specific exercises. In conclusion, moderate intensity exercise is beneficial in ALS, helping in avoiding deconditioning and muscle atrophy resulting from progressive inactivity.
\end{abstract}

Key Words: Amyotrophic Lateral Sclerosis, neurorehabilitation, endurance training, isometric exercise

Eur J Transl Myol 28 (1): 132-140, 2018

Motor neuron diseases (MND) include a heterogeneous spectrum of inherited and sporadic clinical disorders of the upper motor neurons (UMN), lower motor neurons (LMN) or a combination of both. MND are characterized by progressive degeneration and loss of motor neurons in the spinal cord, brain stem, or motor cortex, and manifest clinically as muscle weakness, atrophy, and corticospinal tract signs and symptoms in various combinations. ${ }^{1}$ Differently from most of neuromuscular pathologies, where a moderate consistent exercise is recommended, physical activity in ALS is still much debated. In the past, patients were frequently suggested to avoid regular exercise in order to preserve muscle strength. ${ }^{2}$ In a recent study, it has been demonstrated that resistance and endurance exercise are safe to be performed without any worsening of outcomes in ALS patients. ${ }^{3}$ However, inactivity can involve cardiovascular deconditioning and atrophy from disuse that further superimpose the weakness caused by the ALS disease itself. ${ }^{3}$ If the reduced level of motor activity persists, ALS patients can develop severe deconditioning, muscle atrophy and tendon retractions that cause contractures, pain and higher difficulty in performing daily activities. Current clinical management for people with ALS is primarily individualized rehabilitation that may or may not include strengthening or aerobic exercise prescription. The continued lack of robust evidence regarding the efficacy and benefits of exercise in people with ALS may influence the availability, accessibility and quality of rehabilitation services provided for this group. Exercise, when prescribed appropriately, may be physically and psychologically important for people with ALS, especially in the earlier stages of the disease. Currently, rehabilitation of ALS patients is primarily individualized and sometimes may include strengthening and aerobic exercise. ${ }^{4}$ However, the lack of evidence of impact and 
efficacy of exercise in maintaining motor neuron integrity, motor function, and survival in ALS influences the availability, accessibility and quality of treatments. ${ }^{4}$ Previous animal studies had provided good basic scientific information about the effects of endurance exercise training at moderate intensity showing a higher survival and a reduction of motor neuron degeneration in mice with SOD-1-induced ALS. ${ }^{5,6}$ Recent SOD-1 ALS mice study, have proposed molecular basis for the differential effects of specific exercise type and intensity in ALS; in particularly, the switching to glycolytic muscle metabolism and the activation of the fast motor unit induced by well-designed exercise programs (swimming), may provide synergistic beneficial effects for ALS patients. ${ }^{7,8}$ Considering that, in animal studies the neurons controlling fast fibers were noted to degenerate early in the course of ALS, other authors advise to minimize stress on fast twitch muscle fibers. ${ }^{9,10}$ However, in humans, findings are more heterogeneous. In humans, an association between physical activity and ALS was postulated by some studies; recently a
European Population-Based Case-Control. ${ }^{11}$ Study concluded that physical activity is not a risk and may eventually be protective against the disease. Another review concluded that current evidence for physical activity as a risk factor in MND is limited. ${ }^{12}$ Several authors have attempted to assess functional improvements after moderate exercise highlighting short-term benefits in producing a slower rate of decline in terms of endurance, spasticity and motor deterioration, but not in muscle strength.,10,13-15 On the other hand, other studies lay out some concerns on the exercise programme that should consider the underlying pathophysiology of ALS, avoiding high-intensity exercise that can stress the fast-twitch muscle fibers and being focused on reduction of spasticity, strengthening of weak muscles, and minimization of fatigue. ${ }^{10}$ Overall, aerobic exercises, early and at mild to moderate intensities, seem to be beneficial in muscle strengthening and cardiovascular exercise can help maintain function, prevent further deconditioning and atrophy and not adversely affect the progression of the disease. ${ }^{3-10}$

Table 1. Baseline characteristics of exercise programme group (ALS-EP) and standard neuromotor rehabilitation treatment group (ALS-SNT). ALSFS-R, Amyotrophic Lateral Sclerosis Functional Scale-revised; FIM, Functional Independence Measure; FSS, Fatigue Severity Scale; MRC sum score, Medical Research Concil sum score.

\begin{tabular}{lll}
\hline & $\begin{array}{l}\text { ALS-EP n=23 } \\
\text { mean } \pm \text { SD }\end{array}$ & $\begin{array}{l}\text { ALS-SNT n=15 } \\
\text { mean } \pm \text { SD }\end{array}$ \\
\hline Age, yr & $61.6 \pm 10.6$ & $59.8 \pm 14.7$ \\
Gender, F/M & $10 / 13$ & $4 / 10$ \\
Onset,Spinal/Bulbar & $22 / 1$ & $15 / 0$ \\
Disease duration, mo & $30.2 \pm 11.8$ & $30.3 \pm 6.7$ \\
ALSFRS-R & $36.1 \pm 4.71$ & $34.5 \pm 3.6$ \\
ALSFRS-R, Bulbar subscore & $9.89 \pm 1.7$ & $9.73 \pm 1.7$ \\
Rate of progression & $0.4 \pm 0.18$ & $0.4 \pm 0.05$ \\
FIM & $79.73 \pm 6.1$ & $78.0 \pm 8.3$ \\
FSS & $5.4 \pm 0.27$ & $5.4 \pm 0.2$ \\
Resting heart rate, bpm & $80.52 \pm 5.4$ & $78.80 \pm 8.47$ \\
Oxygen Consumption, ml/min/kg & $15.36 \pm 2.0$ & $12.67 \pm 1.60$ \\
Ventilation, L/min & $25.95 \pm 5.2$ & $24.49 \pm 5.62$ \\
MRC Sum Score & $40.26 \pm 2.45$ & $39.87 \pm 4.24$ \\
Right Biceps Muscle, $\mathrm{kg} / \mathrm{min}$ & $66.96 \pm 24.95$ & $73.13 \pm 16.99$ \\
Left Biceps Muscle, $\mathrm{kg} / \mathrm{min}$ & $70.65 \pm 19.53$ & $80.27 \pm 14.60$ \\
Right Tibial Muscle, $\mathrm{kg} / \mathrm{min}$ & $77.11 \pm 34.92$ & $57.93 \pm 27.74$ \\
Left Tibial Muscle, $\mathrm{kg} / \mathrm{min}$ & $77.21 \pm 36.11$ & $60.80 \pm 32.30$ \\
Six Minute Walk Test, mt & $265.17 \pm 81.37$ & $236.26 \pm 76.26$ \\
& & \\
\hline
\end{tabular}


Physical training in ALS: A pilot, randomized, controlled study

Eur J Transl Myol 28 (1): 132-140, 2018

Table 2. The cardiovascular and metabolic data of ALS-EP patients; I (initial), F (final); HR 65\% (heart rate corresponded to $65 \%$ for aerobic training); RHR (Resting heart rate bpm); VO2 (Oxygen Consumption, $\mathrm{ml} / \mathrm{min} / \mathrm{kg}) ; 6 \mathrm{MW}$ (Six Minute Walk Test, mt); MRCss (MRC Sum Score)

\begin{tabular}{|c|c|c|c|c|c|c|c|c|c|c|}
\hline ALS-EP & age & HR $65 \%$ & RHR I & RHR F & $\mathrm{VO}_{2} \mathrm{I}$ & $\mathrm{VO}_{2} \mathrm{~F}$ & $6 \mathrm{MW} \mathrm{I}$ & $6 \mathrm{MW} \mathrm{F}$ & MRCss I & MRCSs F \\
\hline 1 & 71 & 95 & 88 & 81 & 13 & 8,1 & 152,5 & 170,5 & 42 & 52 \\
\hline 2 & 54 & 110 & 83 & 78 & 18,1 & 15,4 & 392 & 530 & 38 & 50 \\
\hline 3 & 65 & 100 & 89 & 82 & 14,1 & 9 & 294,5 & 298,75 & 41 & 49 \\
\hline 4 & 72 & 95 & 85 & 79 & 12,9 & 12,2 & 316 & 458 & 42 & 48 \\
\hline 5 & 53 & 110 & 81 & 77 & 16,4 & 8,6 & 48 & 68 & 41 & 49 \\
\hline 6 & 71 & 95 & 78 & 73 & 15,4 & 9,5 & 216 & 267 & 36 & 41 \\
\hline 7 & 65 & 100 & 72 & 68 & 17,3 & 11,4 & 256 & 320,5 & 34 & 42 \\
\hline 8 & 68 & 100 & 74 & 67 & 12,8 & 9,3 & 315 & 395 & 40 & 48 \\
\hline 9 & 64 & 100 & 78 & 71 & 15,6 & 11,5 & 375 & 400 & 40 & 50 \\
\hline 10 & 59 & 105 & 84 & 78 & 16,7 & 13,8 & 340 & 557 & 38 & 42 \\
\hline 11 & 73 & 95 & 88 & 81 & 15,9 & 13,1 & 325 & 455 & 41 & 52 \\
\hline 12 & 63 & 100 & 83 & 78 & 17,5 & 12,5 & 238 & 280 & 38 & 47 \\
\hline 13 & 26 & 125 & 89 & 82 & 17,8 & 13,6 & 275 & 340 & 42 & 49 \\
\hline 14 & 67 & 100 & 85 & 79 & 18,3 & 14,3 & 245 & 325 & 45 & 52 \\
\hline 15 & 62 & 105 & 81 & 77 & 19,5 & 14,4 & 135 & 225 & 41 & 48 \\
\hline 16 & 49 & 110 & 78 & 73 & 14,6 & 10,1 & 305 & 405 & 42 & 49 \\
\hline 17 & 57 & 100 & 72 & 68 & 15,2 & 13,1 & 225 & 275 & 41 & 48 \\
\hline 18 & 60 & 105 & 74 & 67 & 13,4 & 11,4 & 325 & 375 & 42 & 49 \\
\hline 19 & 61 & 105 & 78 & 71 & 13,2 & 9,1 & 225 & 275 & 38 & 45 \\
\hline 20 & 74 & 95 & 84 & 78 & 14,6 & 8 & 260 & 325 & 42 & 49 \\
\hline 21 & 63 & 105 & 77 & 73 & 14 & 11,2 & 325 & 350 & 38 & 46 \\
\hline 22 & 71 & 95 & 76 & 69 & 13,2 & 7,9 & 325 & 440 & 42 & 50 \\
\hline 23 & 50 & 110 & 75 & 71 & 13,7 & 8,9 & 186 & 210 & 42 & 51 \\
\hline
\end{tabular}

However, this evidence is not sufficiently detailed to recommend a specific treatment and there is complete lack of randomised clinical trials examining aerobic training in ALS patients. The present study was aimed at evaluating the effect of a moderate exercise programme based on aerobic training and isometric sub-maximal contractions of muscles, on changes in muscle strength, fatigue and cardiovascular parameters. This programme was designed to strengthen the weak muscles, facilitate musculoskeletal resistance and minimize fatigue to prevent deconditioning and atrophy not directly caused by the disease.

\section{Materials and Methods}

\section{Participants}

Forty-six patients admitted to the Foundation San Camillo Hospital for rehabilitation were consecutively enrolled in this study. All patients fulfilled the revised El Escorial criteria for clinically probable, laboratory- supported probable, or definite ALS. ${ }^{20}$ Inclusion criteria included: sporadic or familial ALS; mild to moderate disabilities assessed by Sinaki-Mulder stages I-II-III. ${ }^{19}$ Exclusion criteria were as follows: history of other neurological diseases, lost ability to walk (any assistive device was allowed), mechanical ventilation, heart or respiratory failure, metabolic disorders, any severe active neuropsychological illness making a patient unable to understand and perform the instructions. All patients included in our study were taking riluzole. None carried the SOD1 mutation. Before being enrolled in the study, an evaluation of oxygen consumption was carried out. No participants reported dyspnoea or respiratory insufficiency as assessed by forced vital capacity and nocturnal oximetry. Patients were assigned randomly to two groups: one group (26 patients, 9 females, mean age $61.6 \pm 10)$ underwent a specific exercise programme (ALS-EP) and the second group (20 patients, 8 females, mean age $59.2 \pm 14$ ) followed the standard neuromotor 


\section{Physical training in ALS: A pilot, randomized, controlled study}

Eur J Transl Myol 28 (1): 132-140, 2018

rehabilitation treatment (ALS-SNR). Both groups had no significant difference in their age, gender, time from disease onset, percentage of bulbar onset, rate of progression and disability at time of inclusion in the study (Table 1). The rate of progression of ALS was calculated by dividing the ALSFRS-R total score by symptom duration (months) and seems to represent an independent and robust prognostic biomarker for ALS. ${ }^{21}$ Functional disability was assessed by the revised ALS Functional Rating Scale (ALSFRS-r) and the bulbar involvement was scored using the part of the ALSFRS-r assessing speech, salivation and swallowing. The experimental procedure was approved by the local Ethics Committee for medical research, and all participants gave informed written consent.

\section{Exercise Protocol}

The exercise protocol has been set based on the recommendations of the American College of Sports Medicine (ACSM). ${ }^{22}$ The specific exercise programme consisted of a series of exercises that included an individualized progressive training of muscular strengthening and aerobic endurance, under the supervision of two experienced physical therapists. To be sure to avoid muscle damage, we have chosen the submaximal isometric contraction to strengthen the weak muscles with a score of 3,4- or 4 as measured by the Medical Research Council scale (MRC). The repetitions were 3 for each bilateral muscle segment and were performed for a time interval, determined by calculating $80 \%$ of the time of maximum contraction; each repetition was separated by 30 seconds of rest. The exercise was carried out using rubber bands with a sequential system of progressive resistance and followed by stretching. The use of elastic bands reduces the risk of orthopedic and muscular injuries. The series of exercises was performed daily for 5 weeks. Aerobic exercise was performed to a sub-maximal intensity of $65 \%$ of maximum heart rate adjusted for age, with a duration of 15-20 minutes (in Tables $2 \mathrm{a}-2 \mathrm{~b}$ the HR corresponding to $65 \%$ are summarized for each patient). Based on the weakness pattern and the level of disability of each patient, we chose to use a cycle ergometer, ergometry arm-leg and/or treadmill. Exercises were carried out with continuous monitoring of heart rate and saturimetry. The series of exercises was performed daily for 5 weeks, and overall each exercise session lasted about one hour. The second group of patients (ALS-SNR) followed a 5-week programme of standard neurorehabilitation, consisting of one-hour sessions of stretching, active mobilization and general muscle reinforcement; this last treatment has been set within the limits of patient reported fatigue and avoiding eccentric and concentric contractions. Furthermore, all patients underwent speech, occupational and psychological therapies, based on individual clinical and functional profiles.

\section{Clinical assessment}

All patients were assessed at the beginning and at the end of treatment by two evaluators with experience in neurorehabilitation, blinded to the treatment patients received. Functional autonomy was assessed by the

Table 3. The cardiovascular and metabolic data of ALS-SNP patients

\begin{tabular}{|c|c|c|c|c|c|c|c|c|c|c|}
\hline $\begin{array}{l}\text { ALS- } \\
\text { SNT }\end{array}$ & age & HR $65 \%$ & RHR I & RHR F & $\mathrm{VO}_{2} \mathrm{I}$ & $\mathrm{VO}_{2} \mathrm{~F}$ & $6 \mathrm{MW} \mathrm{I}$ & $6 \mathrm{MW} \mathrm{F}$ & MRCss I & MRCSs F \\
\hline 1 & 54 & 110 & 87 & 85 & 13 & 22,4 & 157,5 & 158,5 & 43 & 46 \\
\hline 2 & 65 & 100 & 91 & 88 & 13,5 & 21 & 157,5 & 158,9 & 42 & 45 \\
\hline 3 & 57 & 110 & 83 & 79 & 13,9 & 19,3 & 172,3 & 172 & 38 & 41 \\
\hline 4 & 58 & 105 & 86 & 84 & 10,4 & 38 & 242 & 242 & 48 & 51 \\
\hline 5 & 57 & 110 & 85 & 81 & 12,4 & 21 & 158 & 157,5 & 42 & 45 \\
\hline 6 & 33 & 120 & 81 & 80 & 9,4 & 23,7 & 250 & 250 & 41 & 43 \\
\hline 7 & 60 & 105 & 82 & 80 & 12 & 24 & 335 & 337 & 39 & 40 \\
\hline 8 & 63 & 95 & 56 & 70 & 15 & 30 & 150 & 170 & 38 & 39 \\
\hline 9 & 29 & 125 & 72 & 75 & 15,1 & 30,2 & 305 & 307 & 44 & 45 \\
\hline 10 & 45 & 115 & 81 & 78 & 18 & 26,6 & 280 & 290 & 38 & 38 \\
\hline 11 & 76 & 95 & 78 & 79 & 11,9 & 17 & 168 & 167 & 36 & 36 \\
\hline 12 & 79 & 90 & 72 & 71 & 11 & 28,2 & 188 & 188 & 36 & 36 \\
\hline 13 & 73 & 95 & 78 & 79 & 12,8 & 26,7 & 275 & 280 & 40 & 41 \\
\hline 14 & 75 & 95 & 78 & 76 & 9,9 & 17 & 270 & 271 & 36 & 36 \\
\hline 15 & 64 & 100 & 72 & 69 & 12,6 & 22,3 & 395 & 398 & 38 & 38 \\
\hline
\end{tabular}




\section{Physical training in ALS: A pilot, randomized, controlled study}

Eur J Transl Myol 28 (1): 132-140, 2018

Functional Independence Measure (FIM). The sixminute walk test (6MWT) was used to identify gait speed thresholds supporting functional ambulation and the effects of fatigue on patients' walking capacity. Muscle strength was evaluated by the MRC Muscle Grading Scale and a dynamometer able to quantify muscle strength reliably (CIT Technics BV, Groningen, the Netherlands), with a reset button and a curved applicator. The muscular strength was assessed by the mean value of three consecutive muscular contractions of Deltoid, Biceps, Wrist extensor, Ileopsoas, Quadriceps femoris, Tibialis anterior. Positions of patient and instrument were standardized as described by Van der Ploeg et al. ${ }^{23}$ The total MRC sum score (MRCSS), ranging from 0 (total paralysis) to 60 (normal strength), was calculated by the sum of the MRC score of the 6 muscles on both sides, each muscle graded from 0 to 5 . Gas analysis was undertaken using the ergometer metabolic system Fitmate (Cosmed@), Rome, Italy) (24-25) following a 5min warm-up at $30-\mathrm{W}$ cycling and a pedalling cadence up to achieve $65 \%$ of the predicted maximum heart rate (HRmax) for 10 minutes. The HRmax was predicted from 220 beats min - age. Speed, resting heart rate and during exercise, ventilation and oxygen uptake were monitored, and the linear regression relating to HR and the volume of $\mathrm{O} 2(\mathrm{VO} 2)$ at submaximal work was extrapolated. Sub maximal oxygen consumption (VO2submax) was measured by Fitmate, using the following formula: VO2submax $(\mathrm{ml} / \mathrm{min} / \mathrm{kg})=(\mathrm{HR} \times$ $\mathrm{SV}) \times[\mathrm{a}-\mathrm{vO} 2]$, where $\mathrm{SV}$ is stroke volume, and a-v O2 is arteriovenous oxygen difference in the oxygen content of the blood between the arterial blood and the venous blood. VO2submax was measured at the same load of exercise before the aerobic training and at the end of the study. Finally, the Fatigue Severity Scale (FSS) was administered to know the degree of fatigue and its effect on the activities of the patients. All patients performed a creatine kinase $(\mathrm{CK})$ test to evaluate potential muscle damage as a consequence of level and intensity of physical exercise. No patients showed altered CK values before and after treatment.

\section{Statistical analysis}

To test for a statistically significant effect of exercise over the treatment period and whether this effect changed in relation to type of treatment, a mixed model, with group (exercise vs. control) and time (T0 vs. T1) as factors, was applied to the FSS and FIM scales, the cardio-respiratory and muscular measurements. ${ }^{26,27}$ Model assumptions were verified through analysis of residuals. In case of heterogeneity, a variance covariate structure was introduced into the model and data were transformed with Box-Cox method in case of evidence of non-normality. Homogeneity of the two groups at T0 was assessed in relation to the ALSFRSr scale, duration of disease, progression rate and age using either the t-test or the non-parametric Mann-Witney test according to whether the data were found Gaussian or not. Correlation between outcome measures was tested with either Pearson or non-parametric Kendall test. Bonferroni correction was then used. ${ }^{28}$ A Shapiro-Wilk test was performed to test for normality of the data. Data were tested at $95 \%$ significance level.

Table 4. Functional and cardiopulmonary changes after training.

\begin{tabular}{lll}
\hline & $\begin{array}{l}\text { ALS-EP n=23 } \\
\text { mean } \pm \text { SD }\end{array}$ & $\begin{array}{l}\text { ALS-SNT } \mathbf{n}=\mathbf{1 5} \\
\text { mean } \pm \text { SD }\end{array}$ \\
\hline FIM & $4.56 \pm 1.16^{* \circ}$ & $1.8 \pm 1.37^{*}$ \\
FSS & $1.29 \pm 0.21^{*}$ & $-0.21 \pm 0.16$ \\
Resting heart rate, bpm & $-5.7 \pm 1.29$ & $-0.53 \pm 4.47$ \\
Oxygen Consumption, $\mathrm{ml} / \mathrm{min} / \mathrm{kg}$ & $-4.16 \pm 1.71^{* \circ}$ & $0.10 \pm 1.53$ \\
Ventilation, L/min & $-3.59 \pm 4.73$ & $0.34 \pm 4.64$ \\
MRC Sum Score & $7.83 \pm 1.80^{* \circ}$ & $1.53 \pm 1.13$ \\
Right Biceps, kg/min & $9.26 \pm 13.17 * \circ$ & $-0.73 \pm 1.10$ \\
Left Biceps, kg/min & $8.1810 .09 \pm$ & $-0.54 \pm 1.35$ \\
Right Tibial, kg/min & $9.28 \pm 12.96$ & $-0.13 \pm 1.46$ \\
Left Tibial, kg/min & $8.44 \pm 17.27$ & $-1.00 \pm 1.41$ \\
Six Minute Walk Test, mt & $71.56 \pm 50.72$ & $2.90 \pm 5.48$ \\
\hline
\end{tabular}

* Significant effect of time, $\mathrm{p}<0.05{ }^{\circ}$ Significant difference between groups, $\mathrm{p}<0.05$ 


\section{Physical training in ALS: A pilot, randomized, controlled study}

Eur J Transl Myol 28 (1): 132-140, 2018

\section{Results}

At T0, both groups had the same degree of disability evaluated by the ALS-FRSr scale $(\mathrm{p}=0.27)$. Five bulbar onset patients were in the ALS-SNR group and one was in the ALS-EP group. Eight patients dropped out and 38 completed the study: the remaining patients were 23 attributable to the group of the specific exercise protocol and 15 to the group of standard rehabilitation. The cardiovascular and metabolic data of ALS-EP and ALSSNP groups are summarized in Table 3. Significant differences in clinical and functional assessment are summarized in Table 4. The FIM scale was found correlated with Ventilation during exercise $(\mathrm{r}=0.25, \mathrm{p}<0.01)$, resting HR $(\mathrm{r}=-0.20, \mathrm{p}=0.01)$, Right Biceps strength $(\mathrm{r}=0.24 ; \mathrm{p}<0.01)$ and the six-minute walk test measurements $(r=0.23, p<0.01)$. The FSS scale correlated with the Oxygen Consumption $(\mathrm{r}=0.26, \mathrm{p}<0.01)$, resting HR $(\mathrm{r}=0.29, \mathrm{p}<0.01)$, Right Biceps and Tibial strength $(\mathrm{r}=-0.21, \mathrm{p}=0.01, \mathrm{r}=-$ $0.19, \mathrm{p}=0.02$, respectively), the MRC Sum Score ( $\mathrm{r}=-$ $0.28, \mathrm{p}<0.01)$ and the Six Minute walk test ( $\mathrm{r}=-$ $0.27, \mathrm{p}<0.01)$.

\section{Discussion}

The positive effects of exercise in healthy people are, in general, well documented and have been largely demonstrated. Accordingly, physical inactivity is associated with increasing risk of many adverse health conditions. ${ }^{29}$ This is more relevant in the process of aging or some neuromuscular disorders where a progressive degradation of the neuromuscular system manifests by a steady decline in muscle mass and strength. In fact, at the level of the single motor unit, aging and disease can be associated with muscle atrophy. Furthermore, recent data show that physical activity promotes reinnervation of muscle fibers in aging. ${ }^{30-35}$ In ALS, conflicting results have weakened the role of physical exercise, though a growing number of data indicates the safe and the beneficial effects of physical activity. ${ }^{3,36,37}$ Among the benefits, we found an overall improvement of the score on functional independence scale in all patients, independently of the type of exercise conducted (specific programme or standard neuromotor rehabilitation treatment). In addition, improvements in muscle power (expressed by the MRC scale), oxygen consumption (measured by VO2submax) and fatigue were specifically observed in the EP group, all hallmarks of a training effect for the specific exercise programme. As for physical activity in general, improvements can potentially reduce the strains of daily living by increasing reserve capacities. ${ }^{38}$ This would allow ALS patients to meet the physiological demands required for activities of daily living more effectively, reflecting an increase of their score on functional independence scale. Examining our combined exercise programme, our purpose was to assess the clinical efficacy of a programme of moderate aerobic and isometric exercises, through an objective assessment of muscle strength, fatigue and cardiovascular parameters, in the early stage of ALS. The specific exercise programme was based on a standardized training consisting of an aerobic workout of moderate entity and muscle contractions. As for the aerobic exercise, treadmill or cycloergometer training is a highly repetitive form of gait training that promotes specific practice and can provide an aerobic training stimulus. Aerobic training can however primarily increase aerobic capacity through cardiorespiratory reconditioning and promote cardiovascular function, improving cardiovagal modulation. Although there are no data on ALS patients, it has been shown that an increase in neuromuscular activity (e.g. repeated exercise), results in a fast-to-slow transition, and that the lack of activity (e.g. resulting from denervation), induces a slow-to-fast transition. ${ }^{8}$ These considerations suggest that aerobic exercise might be the best option for exercise prescription. In parallel to aerobic training, resistance exercises were set accurately, defining the type of contraction, the muscle segments to be reinforced, and the number of repetitions per session. In our study, a submaximal isometric contraction was chosen to avoid muscle damage from mechanical and bioelectrical overload, or metabolic stress, especially to the fast twitch fatigable muscular fibers. During isometric exercise, in fact, the muscle is activated, maintaining a constant length, and reaches a state of maximum tension safely. Recent studies have also shown that isometric exercises can maximize motor unit activity in terms of increase of the firing rates of active motor units and recruitment of other motor units that usually are inactive. $^{39}$ This mechanism can be crucial in strengthening a denervated muscle and could explain the improvement of MRC muscle scale that we observed in our patients after the five-week training programme. In summary, the use of a combined protocol of aerobic and resistance training (below maximal effort) seems to have positive effects in terms of energy and fatigue required to perform activities of daily living, and is associated with increased function and independence. A lower requirement for oxygen by the working muscle during submaximal tasks indicates better walking economy. However, the significant increase in muscular strength expressed by the MRCSS not supported by a change of dynamometric measures of the examined muscles could indicate that the submaximal isometric contractions used were too mild and precautionary. Aerobic training can however primarily increase aerobic capacity through cardiorespiratory reconditioning and promote cardiovascular function, improving cardiovagal modulation. Although there are no data on ALS patients, it has been shown that an increase in neuromuscular activity (e.g. repeated exercise), results in a fast-to-slow transition, and that the lack of activity (e.g. resulting from denervation), induces a slow-to-fast transition. ${ }^{8}$ These considerations suggest that aerobic exercise might be the best option for exercise prescription. In parallel to aerobic training, resistance exercises were set accurately, defining the type of contraction, the muscle segments to 


\section{Physical training in ALS: A pilot, randomized, controlled study}

Eur J Transl Myol 28 (1): 132-140, 2018

be reinforced, and the number of repetitions per session. In our study, a submaximal isometric contraction was chosen to avoid muscle damage from mechanical and bioelectrical overload, or metabolic stress, especially to the fast twitch fatigable muscular fibers. During isometric exercise, in fact, the muscle is activated, maintaining a constant length, and reaches a state of maximum tension safely. Recent studies have also shown that isometric exercises can maximize motor unit activity in terms of increase of the firing rates of active motor units and recruitment of other motor units that usually are inactive. ${ }^{40}$ This mechanism can be crucial in strengthening a denervated muscle and could explain the improvement of MRC muscle scale that we observed in our patients after the five-week training programme. In summary, the use of a combined protocol of aerobic and resistance training (well below maximal effort) seems to have positive effects in terms of energy and fatigue required to perform activities of daily living, and is associated with increased function and independence. A lower requirement for oxygen by the working muscle during submaximal tasks indicates better walking economy. However, the significant increase in muscular strength expressed by the MRCSS not supported by a change of dynamometric measures of the examined muscles could indicate that the submaximal isometric contractions used were too mild and precautionary. suggest that specific rehabilitation programs can counter out the progressive motor deficit of ALS. We believe that moderate exercise can be a reasonable recommendation to establish specific exercise therapy and to improve clinical practice in ALS Centers. Integration with bio molecular data and/or biopsy and a longer exercise programme would be useful to evaluate the effect of exercise on motoneuron efficiency, evidencing cellular and metabolic changes and to verify the effect of rehabilitative intervention on the natural history of the disease.

\section{List of acronyms}

ALS - Amyotrophic Lateral Sclerosis

ALS-EP - specific exercise programme

LMN - lower motor neurons

MND - motor neuron diseases

UMN - upper motor neurons

ALSFS-r - Amyotrophic Lateral Sclerosis Functional Scale- revised

FIM - Functional Independence Measure

FSS - Fatigue Severity Scale

MRCSS - Medical Research Concil sum score.

HR $65 \%$ - heart rate corresponded to $65 \%$ for aerobic training

RHR - Resting heart rate

$\mathrm{VO}^{2}$ - Oxygen Consumption, $\mathrm{ml} / \mathrm{min} / \mathrm{kg}$

$6 \mathrm{MW}$ - Six Minute Walk Test

\section{Author's contributions}

$\mathrm{AM}, \mathrm{FP}$ and CA contributed to the conception and design of the project, the analysis and interpretation of data; in the editing of the work and in the critical review, in the final approval of the version to be published. AL and EG contributed to the conception and design of the project and the acquisition of and interpretation of data. MC and $\mathrm{CG}$ contributed to the analysis and interpretation of data ant to the editing of the work for the final approval of the version to be published.

\section{Acknowledgments and Funding}

Authors thanks for collaboration Simonetta Rossi BSc, Veronica Santoro BSc, Valentina Vianello Bsc, Alfons Baba BSc. Funding: None.

\section{Conflict of Interest}

The authors report no conflicts of interests.

\section{Ethical Publication Statement}

We confirm that we have read the Journal's position on issues involved in ethical publication and affirm that this report is consistent with those guidelines.

\section{Corresponding Author}

Antonio Merico, Via Alberoni, 70, 30126 Venice

Phone Number: 0039 0412207517; Fax number: 0039 0412207129.

E-mail: antonio.merico@ospedalesancamillo.net

E-mails of co-authors

Marianna Cavinato: marianna.cavinato@ ospedalesancamillo.net

Caterina Gregorio: caterina.gregorio@ospedalesancamillo.net

Alessandra Lacatena: alessandralacatena@gmail.com

Elisabetta Gioia: elisabettagioia@live.it

Francesco Piccione: francesco.piccione@ospedalesancamillo.net

Corrado Angelini: corrado.angelini@ospedalesancamillo.net

\section{References}

1. Swash M. Clinical features and diagnosis of amyotrophic lateral sclerosis. In: Brown RH Jr, Meininger V, Swash M, editors. Amyotrophic Lateral Sclerosis. London: Martin Dunitz, 2000: 330 .

2. Coble NO, Maloney FP. Interdisciplinary rehabilitation of multiple sclerosis and neuromuscular disorders. In: Maloney FP, Burks JS, Ringel SP editor(s). Effects of Exercise in Neuromuscular Disease. New York: JB Lippincott, 1985:228-38.

3. Clawson LL, Cudkowicz M, Krivickas et al. A randomized controlled trial of resistance and 


\section{Physical training in ALS: A pilot, randomized, controlled study}

Eur J Transl Myol 28 (1): 132-140, 2018

endurance exercise in amyotrophic lateral sclerosis. Amyotroph Lateral Scler Frontotemporal Degener 2017;30:1-9

4. Dal Bello-Haas V, Florence JM. Therapeutic exercise for people with amyotrophic lateral sclerosis or motor neuron disease. Cochrane Database Syst Rev 2013;5:Art. No.: CD005229

5. Kaspar BK, Frost LM, Christian L et al. Synergy of insulin-like growth factor-1 and exercise in amyotrophic lateral sclerosis. Ann Neurol 2005;57:649-55.

6. Deforges S, Branchu J, Biondi O, et al. Motoneuron survival is promoted by specific exercise in a mouse model of amyotrophic lateral sclerosis. J Physiol 2009;587 (Pt 14):3561-72.

7. Desseille C, Deforges S, Biondi O et al. Specific Physical Exercise Improves Energetic Metabolism in the Skeletal Muscle of Amyotrophic-LateralSclerosis Mice. Front Mol Neurosci 2017;10:33.

8. Grondard C, Biondi O, Pariset $\mathrm{C}$ et al. ExerciseInduced Modulation of Calcineurin Activity Parallels the Time Course of Myofibre Transitions J Cell Physiol 2008;214:126-35.

9. Frey, D, Schneider C, Xu L, et al. Early and selective loss of neuromuscular synapse subtypes with low sprouting competence in motoneuron diseases. J Neurosci 20, 2534-42.

10. Lui AJ, Byl NN. A systematic review of the effect of moderate intensity exercise on function and disease progression in amyotrophic lateral sclerosis. J Neurol Phys Ther 2009;33:68-87.

11. Pupillo E, Messina P, Giussiani G Physical Activity and Amyotrophic Lateral Sclerosis: A European Population-BasedCase-Control Study Ann Neurol 2014;75:708-16

12. Harwood CA, McDermott CJ, Shaw PJ. Physical activity as an exogenous risk factor in motor neuron disease (MND): a review of the evidence. Amyotroph Lateral Scler 2009;10:191-204.

13. Carreras I, Yuruker S, Aytan N, et al. Moderate exercise delays the motor performance decline in a transgenic model of ALS. Brain Res 2010;1313:192-201.

14. Drory VE, Goltsman E, Goldman J, et al. The value of muscle exercise in patients with amyotrophic lateral sclerosis J Neurol Sci 2001;191:133-7

15. Lunetta C, Lizio A, Sansone VA, et al. Strictly monitored exercise programs reduce motor deterioration in ALS: preliminary results of a randomized controlled trial. J Neurol 2016;263:5260.

16. Kilmer DD The effect of a high resistance exercise programme in slowly progressive neuromuscular disease. Arch Phys Med Rehabil 1994;75:560-3.

17. Milner-Brown HS, Miller RG. Muscle strengthening through high-resistance weight training in patients with neuromuscular disorders. Arch Phys Med Rehabil 1988;69:14-9.
18. Cup EH, Pieterse AJ, Ten Broek-Pastoor JM, et al. Exercise therapy and other types of physical therapy for patients with neuromuscular diseases: a systematic review Arch Phys Med Rehabil 2007;88:1452-64.

19. Sinaki M, Mulder DW Rehabilitation techniques for patients with amyotrophic lateral sclerosis. Mayo Clin Proc 1978;53:173-8.

20. Chaudhuri KR, Crump S, al-Sarraj S, et al. The validation of El Escorial criteria for the diagnosis of amyotrophic lateral sclerosis: a clinicopathological study.J Neurol Sci 1995;129 Suppl:11-2.

21. Labra L, Menon P, Byth K, et al. S. Rate of disease progression: a prognostic biomarker in ALS J Neurol Neurosurg Psychiatry 2016;87:628-32

22. Garber CE, Blissmer B, Deschenes MR, et al. American College of Sports Medicine position stand. Quantity and quality of exercise for developing and maintaining cardiorespiratory, musculoskeletal, and neuromotor fitness in apparently healthy adults: guidance for prescribing exercise. American College of Sports Medicine Med Sci Sports Exerc 2011;43:1334-59.

23. Van der Ploeg, Oosterhuis HJ, Reuvekamp J. Measuring muscle strength. J Neurol 1984;231:200-3.

24. Lee JM, Bassett DR Jr, Thompson DL, Fitzhugh EC. Validation of the Cosmed Fitmate for prediction of maximal oxygen consumption. J Strength Cond Res 2011;25:2573-9.

25. Nieman DC, Lasasso H, Austin MD, et al. Validation of Cosmed's FitMate in measuring exercise metabolism. Res Sports Med 2007;15:6775.

26. Faraway JJ. Extending the linear model with R: generalized linear, mixed effects and nonparametric regression models. London (UK): Chapman \& Hall/CRC; 2006.

27. West BT, Welch KB, Galecki AT. Linear mixed models: a practical guide using statistical software. London (UK): Chapman \& Hall/CRC; 2006. doi: 10.1201/b17198.

28. Huberty C.J, Morris J.D. Multivariate Analysis versus Multiple Univariate Analyses. Psychological Bulletin 1989;105:302-8.

29. Booth FW, Roberts CK, Laye MJ. Lack of exercise is a major cause of chronic diseases Compr Physiol. Author manuscript; available in PMC 2014 Nov 23.

30. Deschenes MR. Motor unit and neuromuscular junction remodeling with aging. Curr Aging Sci 2011;4:209-20.

31. Mosole S, Carraro U, Kern H et al; Long-term highlevel exercise promotes muscle reinnervation with age. J Neuropathol Exp Neurol. 2014;73:284-94.

32 Mosole S, Carraro U, Kern H, Loefler S, Zampieri S. Use it or Lose It: Tonic Activity of Slow Motoneurons Promotes Their Survival and Preferentially Increases Slow Fiber-Type 


\section{Physical training in ALS: A pilot, randomized, controlled study}

Eur J Transl Myol 28 (1): 132-140, 2018

Groupings in Muscles of Old Lifelong Recreational Sportsmen. Eur J Transl Myol 2016;26:5972. doi: 10.4081/ejtm.2016.5972. eCollection 2016 Sep 15.

33. Willand MP. Electrical Stimulation Enhances Reinnervation After Nerve Injury. Eur J Transl Myol 2015;25:243-8. doi: 10.4081/ejtm.2015.5243. eCollection 2015 Aug 24. Review.

34. Sajer S. Mobility disorders and pain, interrelations that need new research concepts and advanced clinical commitments. Eur J Transl Myol 2017;27(4):7179. doi: 10.4081/ejtm.2017.7179. eCollection 2017 Dec 5.

35. Power GA, Dalton BH, Gilmore KJ, et al. Maintaining Motor Units into Old Age: Running the Final Common Pathway. Eur J Transl Myol 2017;27(1):6597. doi: 10.4081/ejtm.2017.6597. eCollection 2017 Feb 24

36. Pinto AC, Alves M, Nogueira A, et al. Can amyotrophic lateral sclerosis patients with respiratory insufficiency exercise? J Neurol Sci 1999;169:69-75
37. Lui AJ, PT, Byl NN. A systematic review of the effect of moderate intensity exercise on function and disease progression in amyotrophic lateral sclerosis. J Neurol Phys Ther 2009;33:68-87.

38. Chan CW, Sinaki M. Rehabilitation management of the ALS patient. In: Belsh JM, Schiffman PL, editors. Amyotrophic lateral sclerosis: diagnosis and management for the clinician. Amonk (Ny): Futura Publishing Co., Inc; 1996. p. 315-331.

39. Macko RF, Smith GV, Dobrovolny CL, et al. Treadmill training improves fitness reserve in chronic stroke patients. Arch Phys Med Rehabil 2001;82:879-84.

40. De Luca CJ, Kline JC. Influence of proprioceptive feedback on the firing rate and recruitment of motoneurons. J Neural Eng 2012;9:016007.

Received for publication: January 5, 2018

Revision received: February 14, 2018

Accepted for publication: February 14, 2018 